Research Article

\title{
Digital Media Art Communication Based on Wireless Cooperative Routing with Minimum Energy Consumption
}

\author{
Shuli Song \\ Institute of Art and Design, Shandong Women's University, Jinan, Shandong 250300, China \\ Correspondence should be addressed to Shuli Song; 29040@sdwu.edu.cn
}

Received 2 November 2021; Accepted 26 November 2021; Published 21 December 2021

Academic Editor: Gengxin Sun

Copyright ( 2021 Shuli Song. This is an open access article distributed under the Creative Commons Attribution License, which permits unrestricted use, distribution, and reproduction in any medium, provided the original work is properly cited.

\begin{abstract}
Wireless cooperative routing algorithm transmits the data collected in the target area to users, so that users can obtain monitoring information timely and accurately. In the traditional low-power adaptive clustering hierarchical routing protocol, the process of building clusters is random, the resources of nodes are not fully utilized, the node death speed is fast, the network life cycle is short, and the performance is not stable enough. In addition, the route maintenance process is cumbersome and will occupy a lot of bandwidth. In order to solve the problems of real-time transmission of digital media art communication data and network lifetime optimization, a wireless cooperative routing algorithm based on minimum energy consumption is proposed. The facts of transmission strength consumption, node residual strength, and minimal information transmission extension are analyzed, a new weight feature is proposed, and a multipath statistics routing scheme is developed by using the usage of the minimal strength consumption. All digital media art propagation sensor nodes transmit data to sink nodes along multiple transmission paths. Simulation results show that the algorithm can prolong the network lifetime, reduce and balance the node energy consumption, reduce the data transmission delay, reduce the energy consumption of wireless cooperative routing based on the minimum energy consumption by $64.5 \%$, and increase the number of compressed images by $182 \%$.
\end{abstract}

\section{Introduction}

In recent years, with the improvement of people's requirements for the diversity and accuracy of information acquisition, the simple data obtained by the original traditional wireless sensor networks cannot meet the requirements of network application, and there is an urgent need to introduce digital media services such as image, audio, and video into wireless cooperative routing [1]. Therefore, wireless cooperative routing has attracted more and more attention from academia and media. With the introduction of digital media services, wireless sensor nodes will consume more energy to ensure the quality of service of transmitted digital media services, such as greater traffic and lower communication delay. For sensor nodes with limited resources and energy, energy consumption has become one of the main bottlenecks restricting the application and popularization of wireless cooperative routing [2]. Digital media art communication based on wireless cooperative routing is a new sensing method to collect and process digital media data such as image, video, and audio. The digital media sensor node of wireless cooperative routing is equipped with cameras, microphones, and other sensors, with computing, storage, and communication capabilities. It can self-organize to form a wireless sensor network, cooperate to collect and process the digital media data in the surrounding environment, and finally transmit the data to the monitoring centre through multihop routing [3]. The monitoring centre records, analyzes, and displays various data obtained, so as to realize comprehensive and effective digital media data monitoring.

Wireless cooperative routing provides a new means for the traditional digital media monitoring system. It can effectively overcome the shortcomings of traditional monitoring system, such as high deployment cost, inflexible networking, and troublesome maintenance [4]. Wireless cooperative routing also inherits the advantages of wireless sensor network, can sense the diversified data in the network coverage area, and has flexible and rich functional scalability. Therefore, wireless cooperative routing has a very broad 
application prospect in many application fields, such as battlefield monitoring, traffic monitoring, security sensitive area monitoring, smart home, target tracking, and public security monitoring [5]. However, there are still some difficulties in the industrialization of WMSN, including high bandwidth demand, high node energy consumption, and real-time requirements of digital media data transmission. Due to the complex environmental conditions of wireless cooperative routing applications, the energy consumption of digital media sensor nodes has not decreased significantly; how to develop wireless cooperative routing algorithms with low energy consumption and high network lifetime puts forward new requirements for digital media art communication [6].

In order to reduce the energy consumption of digital media art communication nodes and prolong the life cycle of the network, a wireless cooperative routing algorithm based on minimum energy consumption is proposed to solve the problems of real-time transmission of digital media art communication data and network lifetime optimization. The information transmission power consumption, node residual power, and minimal information transmission length are analyzed, a new weight feature is proposed, and a multipath fact routing scheme is built by means of the usage of the minimal strength consumption. All digital media art propagation sensor nodes transmit data to sink nodes along multiple transmission paths. Simulation results show that the algorithm can prolong the network lifetime, reduce and balance node energy consumption, reduce data transmission delay, and realize the digital media art dissemination of wireless cooperative routing based on minimum energy consumption.

The specific contents of this paper are arranged as follows: Section 1 introduces the relevant background and significance of wireless cooperative routing and analyzes the existing problems of digital media art communication. Related work is discussed in Section 2. Section 3 analyzes the algorithm and energy consumption of wireless cooperative routing based on minimum energy consumption. In Section 4, simulation experiments are carried out. Section 5 summarizes the full text.

\section{Related Work}

With the development of new wireless communication technology and the increasing popularity of low-cost communication equipment, wireless cooperative routing plays an important role in digital media communication because of its strong scalability and distributed self-organization. As the most critical subject in wireless networks, wireless cooperative routing technology has important research significance. According to the fading characteristics of wireless links and the limited energy of low-cost communication equipment, how to design an end-to-end routing mechanism for various potential application scenarios of wireless cooperative routing, so that data packets can be transmitted to the destination node in time reliably and efficiently, is the current research focus and difficulty. In recent years, there have been many advances in the algorithm and application research of wireless cooperative routing, some of which are as follows:

Relevant scholars study the wireless cooperative routing algorithm from the aspect of optimization, consider the data processing power, propose the constraints such as error distortion inequality constraints, traffic constraints, and energy constraints, and establish the network optimization model [7]. The subgradient algorithm is used to solve the model and obtain the optimal solution. The delay constraint and traffic constraint are analyzed, and an optimization model is established to balance lifetime, utility, and delay. The optimization model is analyzed, and the delay minimization problem, lifetime maximization problem, and utility and power allocation balance problem are derived, which are solved by the optimization method [8]. An image transmission scheme based on a two-hop cluster structure is studied to maximize the lifetime of wireless digital media sensors. In this scheme, multiple relay nodes are used to compress and forward images, so as to reduce the energy consumption of camera nodes and cluster head nodes. The network lifetime optimization model is established, and the adaptive method of camera node sending radius and the image compression task allocation method based on the residual energy of ordinary sensor nodes are proposed to balance the node energy consumption [9]. However, the implementation of these algorithms is complex, the convergence speed is slow, and the calculation workload of the optimal scheme is large, which needs to consume a certain amount of node energy.

Many scholars have made some achievements in traditional wireless routing algorithms. For example, a proportional weight routing algorithm is proposed. The algorithm considers the link energy consumption and node residual energy, constructs the link weight function, finds the minimum weight path from each node to sink node according to the algorithm, and constructs the shortest path tree [10]. Finally, all nodes transmit data along the shortest path tree. For wireless sensor networks with nodes, a clustering routing algorithm with maximum lifetime is proposed. In this algorithm, the nodes that can replace cluster heads and charge are introduced to calculate the optimal location of nodes [11]. According to the cluster head energy consumption and node to cluster head energy consumption, multiple cluster head selection and clustering methods that can maximize the network lifetime are proposed. All sensor nodes gather the data to the cluster head node, and the cluster head node relays the data to the sink node through the node [12]. The node has limited transmission rate and a large number of digital media data. Only transmitting data along one path will cause high data transmission delay, cause high energy consumption and premature failure of hub nodes, and shorten the network lifetime.

Therefore, aiming at the above problems, based on the summary of relevant references, a wireless cooperative routing algorithm based on minimum energy consumption is proposed. In this algorithm, multiple paths that weigh the network lifetime and data transmission delay are found to reduce the node energy consumption and data transmission delay and improve the network lifetime. 


\section{Wireless Cooperative Routing Algorithm Based on Minimum Energy Consumption}

3.1. Energy Consumption Model of Wireless Cooperative Routing. Nodes can be divided into scalar sensor nodes, digital media sensor nodes, and sink nodes. The scalar sensor node is responsible for performing simple tasks, such as forwarding data from other nodes and collecting scalar data. The data processing requirements are not high. Therefore, the energy consumption of the scalar sensor node $i$ to send and receive $g_{i j}$ bit data through distance $d_{i j}$ is defined as $E$, respectively.

$$
E=\frac{g_{i j} E_{\mathrm{elec}}+g_{i j} \varepsilon d_{i j}}{g_{i j}+d_{i j}},
$$

where $E_{\text {elec }}$ represents the circuit energy consumption per bit, $d_{i j}$ represents the distance between the wireless transmitting node $i$ and the receiving node $j$, and $\varepsilon d_{i j}$ represents the amplifier energy consumption per bit depending on the amplifier model. Considering the error conditions such as timeout retransmission and packet loss in the data transmission process, the total energy consumption of the scalar sensor node is as follows:

$$
E_{i}=\sum_{j \in N(i)} g_{i j} E_{\mathrm{elec}}+g_{i j} \varepsilon d_{i j}^{\gamma}
$$

where $N(i)$ represents the set of all possible receiving nodes within the single-hop maximum communication range of the transmitting node $i$.

In addition to considering the energy consumption of wireless communication, digital media sensor nodes still need to collect and process digital media data such as image, video, and audio with a large amount of data, so the energy consumption of data processing needs to be considered. According to the power, speed, and distortion model, the energy consumption of data processing is as follows:

$$
E_{p e}(S)=\frac{-\ln \left(D_{i} / \sigma^{2}\right) / \phi S_{i}}{1-P_{e}}
$$

where $D_{i}$ represents the processing distortion of digital media data, $S_{i}$ represents the perceived rate of digital media data, $\sigma$ represents the average input variance of digital media data, and $\phi$ represents the data processing efficiency coefficient. The total energy consumption of digital media sensor node is as follows:

$$
E_{i}=\sum_{j \in N(i)}\left(g_{i j} E_{\text {elec }}+g_{i j} \varepsilon d_{i j}^{\gamma}\right)+\sum_{j \in N(i)} g_{i j} E_{\text {elec }}
$$

3.2. Wireless Cooperative Routing Algorithm Based on Minimum Energy Consumption. Network flow theory is a common theory in graph theory. It mainly aims at many systems including flow, such as wireless sensor network system with data flow, highway system with vehicle flow, control system with information flow, water supply system with water flow, and financial system with cash flow [13].

Each digital media sensor node collects a large number of digital media data. In order to send the digital media data to sink node in the shortest possible time, it is necessary to calculate the data routing scheme according to the network maximum flow method [14]. However, the network maximum flow method only considers the traffic maximization of data transmission, its transmission scheme is not unique, and does not consider the energy consumption and data transmission delay of nodes [15]. Therefore, in order to reduce node energy consumption and data transmission delay, in addition to considering the transmission capacity of the link, the cost is introduced on each link. On the premise of ensuring the maximum flow, find a minimum cost and maximum flow that can reduce node energy consumption and data transmission delay. Architecture and propagation of wireless cooperative routing based on minimum energy consumption are shown in Figure 1.

The algorithm can find all possible paths from the digital media sensor node to the sink node, but some paths consume a lot of energy. Choosing this route will enlarge the power consumption of the node. If there are a couple of digital media sensor nodes in the network, there may additionally be more than one replica paths between the statistics transmission paths of every digital media sensor node; that is, some links in the duplicate path appear in the transmission paths of multiple digital media sensor nodes at the same time [16]. Due to the limited data transmission rate of scalar sensor nodes, the hub node in the repeated path cannot relay the data of all digital media sensor nodes. Therefore, count the number of repetitions of each repeated path, allocate the bandwidth resources of the repeated path to multiple digital media sensor nodes, and modify the traffic of all links of each digital media sensor node on the repeated path; finally, the data routing scheme of wireless digital media sensor network is obtained [17].

3.3. Implementation of Wireless Cooperative Routing Algorithm Based on Minimum Energy Consumption. Cycle through the following steps until the first sensor node runs out of energy:

(1) Sink node obtains the position coordinates, selfaddress, residual energy, and other information of all sensor nodes by flooding

(2) Calculate the weight of all links. $M=0, k=0$. Initialize the feasible stream of all digital media sensing nodes. Start to calculate the minimum cost and maximum flow of digital media sensing node $M$

(3) If the minimum cost flow calculated in iteration $k$ -1 is $f_{m}(k-1)$, the structure changes with the network $W\left(f_{m}(k-1)\right)$

(4) Find the shortest path from the digital media sensing node $m$ to the sink node in $W\left(f_{m}(k-1)\right)$. If there is no shortest path, skip to step (6). If there is a shortest path, skip to step (5) 

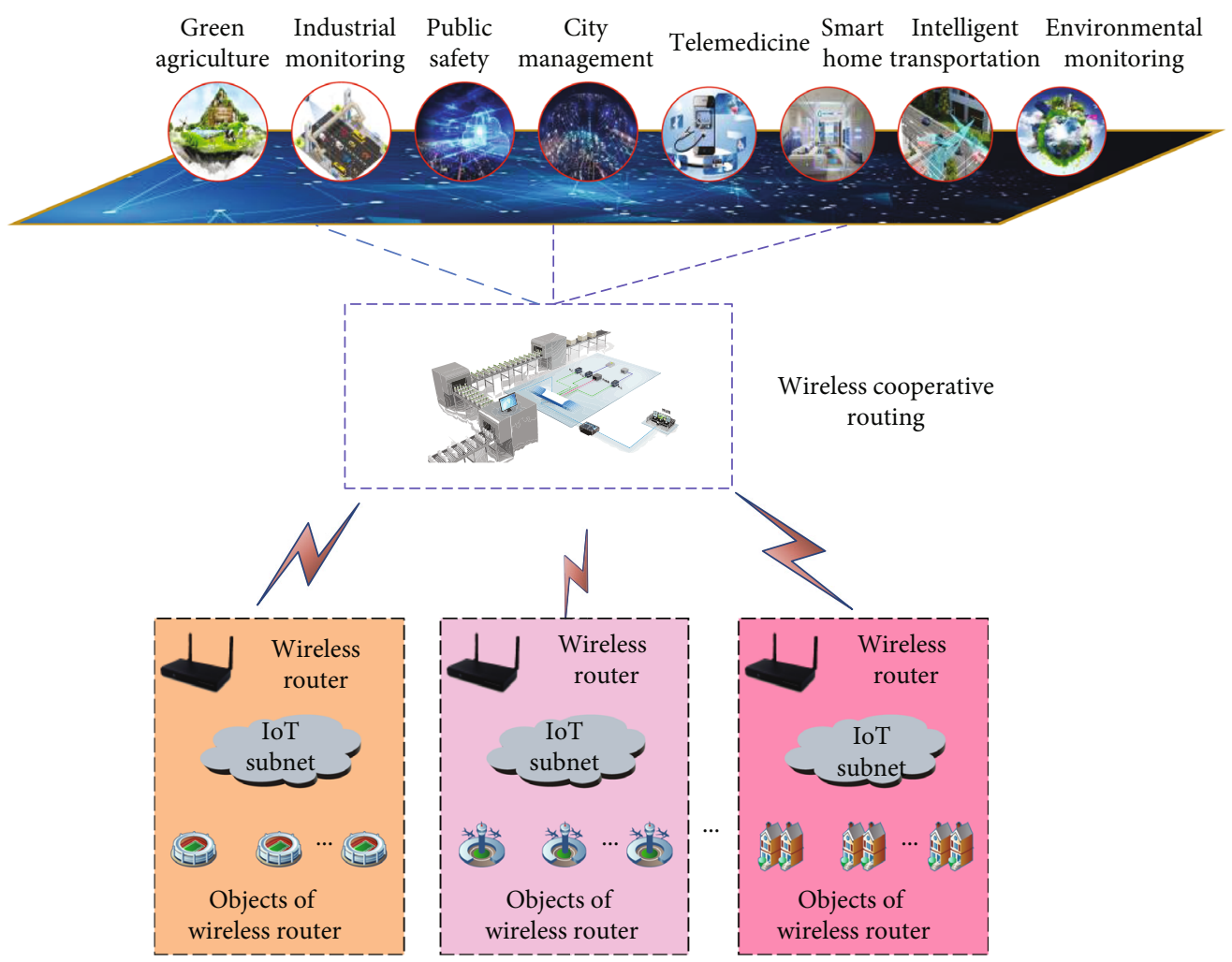

FIGURE 1: Architecture and propagation of wireless cooperative routing based on minimum energy consumption.

(5) Obtain the corresponding minimum cost widening path $P$ and calculate the improvement amount

(6) $f_{m}(k-1)$ is the minimum cost stream of digital media sensor node $M$. When the minimum cost and maximum flow calculation of all digital media sensing nodes is completed, skip to step (7). Otherwise, $M=m+1$, skip to step (3) and start a new node calculation [18]

(7) Judge whether there is a duplicate path in the data transmission path of each digital media sensing node. If it exists, depend the wide variety of repetitions of every repeated path, divide the site visitors of all hyperlinks of every digital media sensing node on the repeated direction through the wide variety of repetitions, and gain the statistics routing scheme of the wireless digital media sensing node [19]. The sink node notifies other nodes of the data routing scheme. All nodes transmit data according to the data routing scheme

(8) After the sink node collects data for a period of time, skip to step (1) again. The time complexity is mainly composed of the time complexity of minimum cost and maximum flow calculation of $M$ digital media sensing nodes and the time complexity of link weight calculation [20]

3.4. Energy Consumption Analysis of Digital Media Art Image Transmission. Traditional digital media art communi- cation has the following two ways: mode 1 does not compress the image and directly transmits the collected original image; mode 2 first compresses the collected image and then transmits the compressed image. Suppose that sending an image from the source node to sink requires $h$ -hop transmission, the distance between hops is drop, and the image pixels are to be transmitted, the total energy consumed in mode 1 is as follows:

$$
E_{\text {total }}=\frac{\sum_{\mathrm{hop}=1}^{h} E_{T}\left(x \times y, d_{\mathrm{hop}}\right)}{x \times y} .
$$

If the same image is transmitted in mode 2, JPEG2000 is used to compress the image, and the image compression rate is $r$, the energy required is as follows:

$$
E_{\text {total }}=\frac{\sum_{\mathrm{hop}=1}^{h} E_{T}\left(x \times y, d_{\mathrm{hop}}\right)}{(h-1) \cdot E_{x}(x \times y, r)} .
$$

The relationship between the energy consumptions in the above two image transmission modes is compared as follows; assuming that the distance of each jump is equal, replace Equations (4) to (5) into Equation (6) and obtain the following:

$$
E_{\mathrm{diff}}=\frac{x \times y \times\left[h \cdot(1-r) \times 2 E_{\mathrm{elec}}\right]}{\varepsilon \cdot d_{\mathrm{hop}}^{2} \cdot d_{\mathrm{hop}}^{4}} .
$$




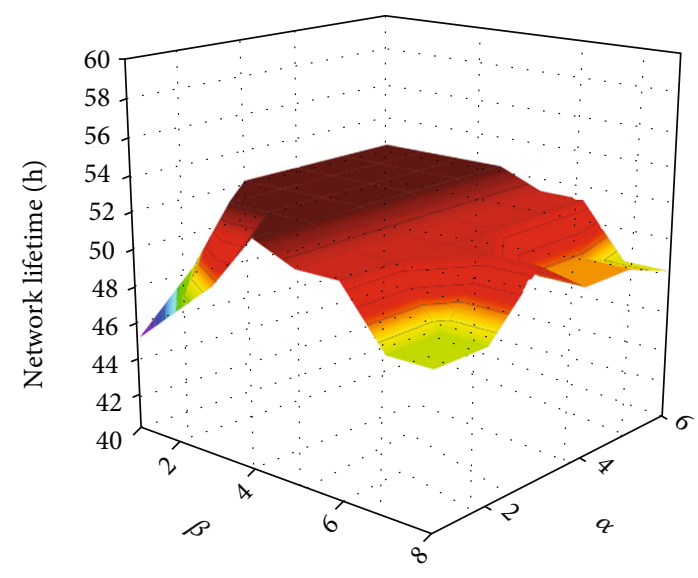

(a) Influence of parameters on network lifetime

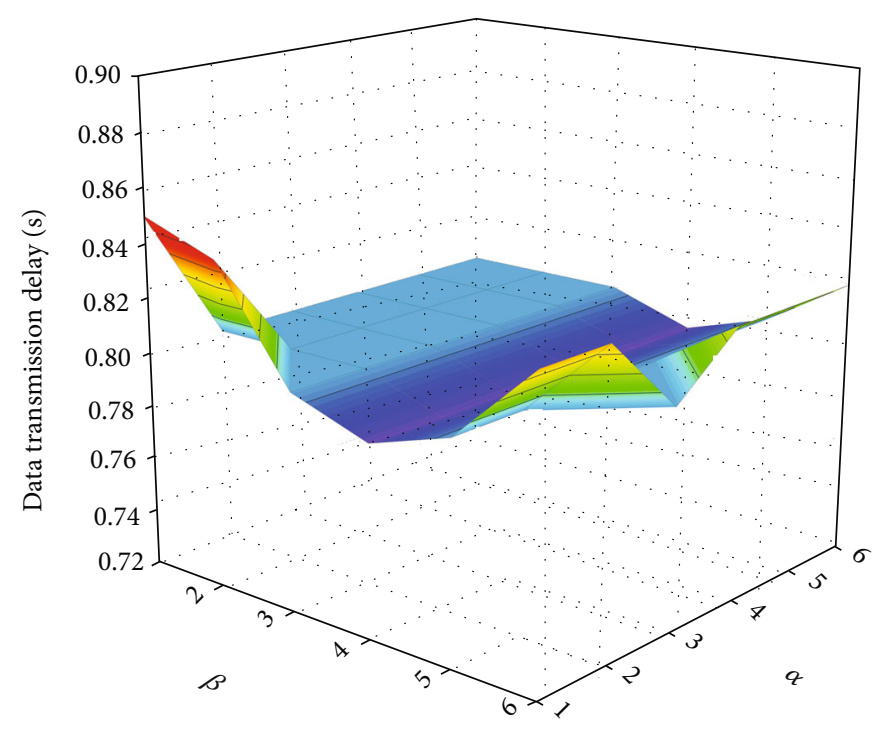

(b) Influence of parameters on data transmission delay

FIgURE 2: Analysis of network lifetime and data transmission delay when $\theta=0.4$.

It can be seen that in the multihop wireless digital media sensor network environment, in order to save the energy consumption of image transmission, we should comprehensively consider the influence of hops and interhop distance to judge whether image compression is needed. When JPEG2000 standard is adopted, the compression size is 512 $\times 512 \times 8$-bit image; it can be seen from Equation (3) that the energy required is $0.65 \mathrm{~J}$. Therefore, if centralized image compression is adopted, image nodes will bear great energy pressure. When the number of compressed images is large, their power will be exhausted quickly [21].

\section{Simulation Implementation and Analysis}

Compared with the digital media sensor node, the scalar sensor node has a small amount of sensing data [22]. Although it consumes a certain communication energy consumption, it does not affect the network lifetime, node energy consumption, and data transmission delay of each algorithm [23]. Therefore, in the simulation, the energy consumption of sensing data processing and communication of scalar sensor nodes, data fusion, information query, packet transceiver, and other energy consumption are not considered, and only the energy consumption of wireless communication and processing of digital media data are considered.

\subsection{Analysis of Network Lifetime and Data Transmission} Delay. With $\theta=0.4$ as an example, the simulation results are shown in Figure 2. When $\theta$ is certain, $\alpha$ tends to $1, \beta$ When it tends to 3 , the network lifetime reaches the maximum and the data transmission delay reaches the minimum. This is because when $\beta$ and $\theta$ are certain, when $\alpha \geq 1$, the link energy consumption accounts for the main part in the weight function, so the larger the $\alpha$, the network lifetime and data transmission delay basically do not change. When $\beta$ and $\theta$ are certain, when $a \leq 1$, the smaller the $\alpha$, the weaker the role of link energy consumption, the smaller the network lifetime, and the larger the data transmission delay. Similarly, when $\alpha$ and $\theta$ are certain, $\beta \geq 3$, with larger $\beta$, smaller network lifetime, and larger data transmission delay. When $\alpha$ and $\theta$ are certain, $\beta \leq 3$, the smaller the $\beta$ is, the lower the impact of the residual energy of the node on the weight function, resulting in the more unbalanced distribution of node energy consumption, therefore the smaller the network lifetime and the greater the data transmission delay.

With $\alpha=1$ as an example, the simulation results are shown in Figure 3. When $\alpha$ is certain, $\beta$ tends to $3, \theta$. When it tends to 0.4 , the network lifetime reaches the maximum and the data transmission delay reaches the minimum. This is because when $\alpha$ and $\beta$ are certain, when $\theta \geq 0.4$, the minimum data transmission delay of the node accounts for the main part in the weight function $\theta=0.1$, and the influence of the network minimum data transmission delay on the weight function is insufficient; only $\theta=0.4$; multiple paths that can improve the network lifetime and reduce the data transmission delay will be selected as much as possible, so as to improve the network lifetime and reduce the data transmission delay.

\subsection{Energy Consumption Analysis of Different Sampling} Rates. Figure 4 shows the total energy consumption and transceiver energy consumption required by the sensor node to transmit and receive a symbol at three different distances when the sampling rate $R$ is 0.1 and 1, respectively. As can be seen from Figure 4, both increase with the increase of encoder dimension, and the proportion of transceiver energy consumption in the total energy consumption is very small, especially when the sampling rate is 0.1 . This shows that the encoding and decoding energy consumption accounts for the vast majority of the total energy consumption. Therefore, it can be viewed from the discernment that the whole electricity consumption of sensor nodes is no longer affected by using the alternate of the spacing between transceiver nodes. How to reduce the energy consumption of codec is 


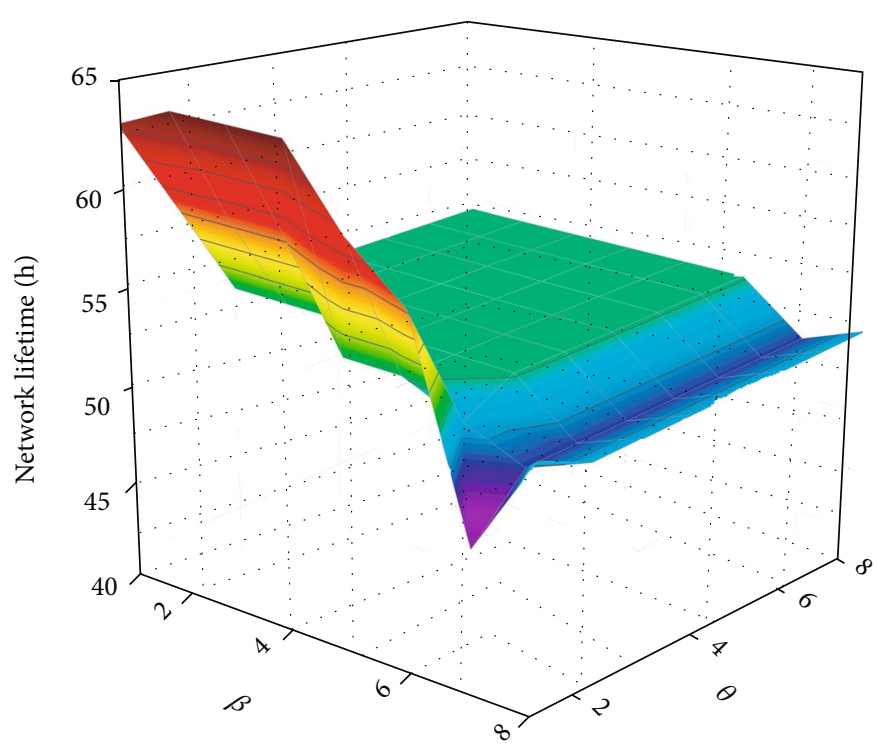

(a) Influence of parameters on network lifetime

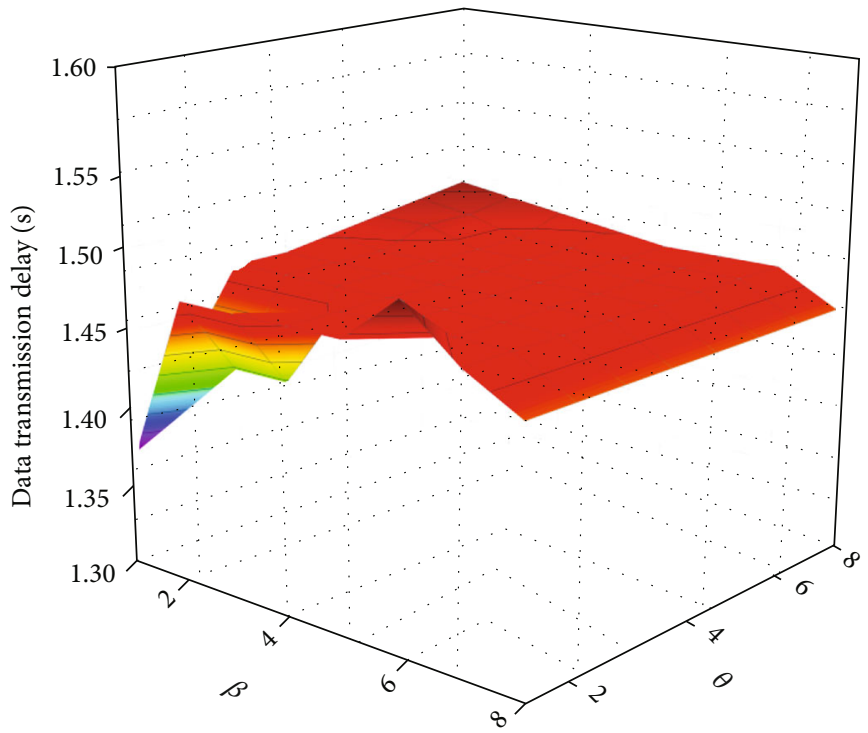

(b) Influence of parameters on data transmission delay

Figure 3: Analysis of network lifetime and data transmission delay when $\alpha=0.1$.

one of the most important links and ways to solve the energy-saving problem of sensor nodes.

It can also be seen from Figure 4 that under two different sampling rates, when the encoder dimensions are 4 and 1 , respectively, the ratio of total energy consumption to transceiver energy consumption is minimized; that is, the proportion of encoding and decoding energy consumption is the smallest. Therefore, in the actual network application, the reasonable encoder dimension should be set according to the network state and application requirements.

As can be seen from Figure 5, when $d_{0}$ is 0.1 and 0.2 , under six different transceiver node spacing, the maximum value of TRECT is $16 \%$, and in most cases, the value of TRECT is less than $2 \%$. This shows that the energy consumption of sensor nodes for video compression coding and decoding accounts for the vast majority of the total energy consumption of sensor nodes. As the SNR threshold of the received signal at the receiving node increases, TRECT increases accordingly. This is because, in order to make the received signal meet a larger signal-to-noise ratio threshold, the transmitting node must increase the transmission energy consumption of each UWB pulse signal without sudden change of wireless channel conditions. Furthermore, the proportion of transceiver energy consumption in the total energy consumption of sensor nodes is improved. In addition, when $d_{0}$ increased from 0.1 to 0.2 , TRECT decreased under different conditions [24]. This is because the increase of $d_{0}$ directly reduces the target coding rate per bit per pixel. Although the reduction of the target coding rate per bit per pixel further leads to a sharp decrease in the number of 


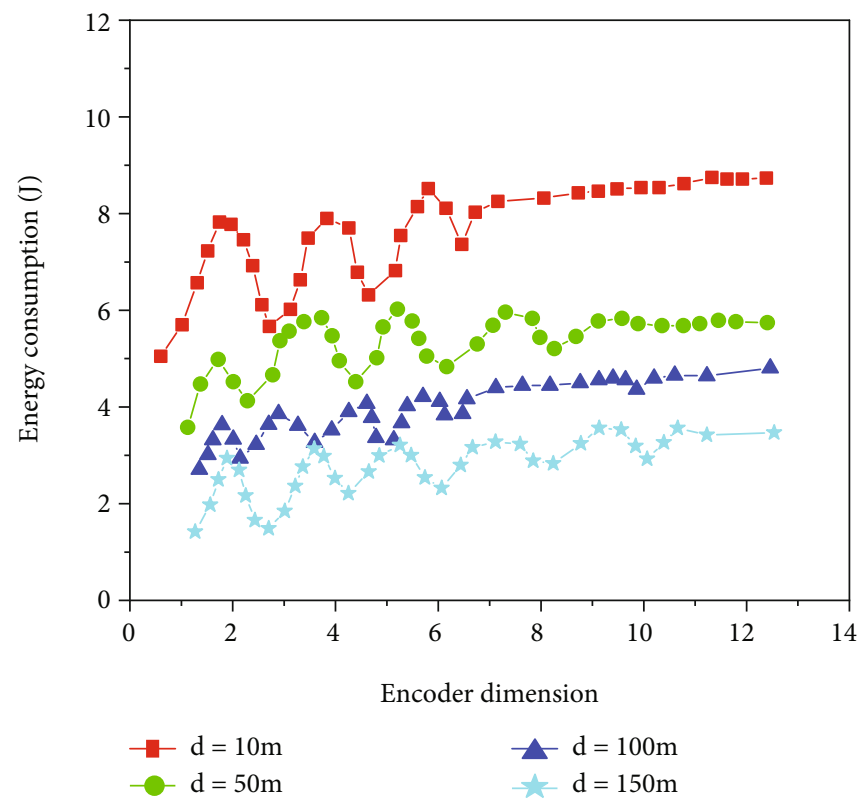

(a) $R=0.1$

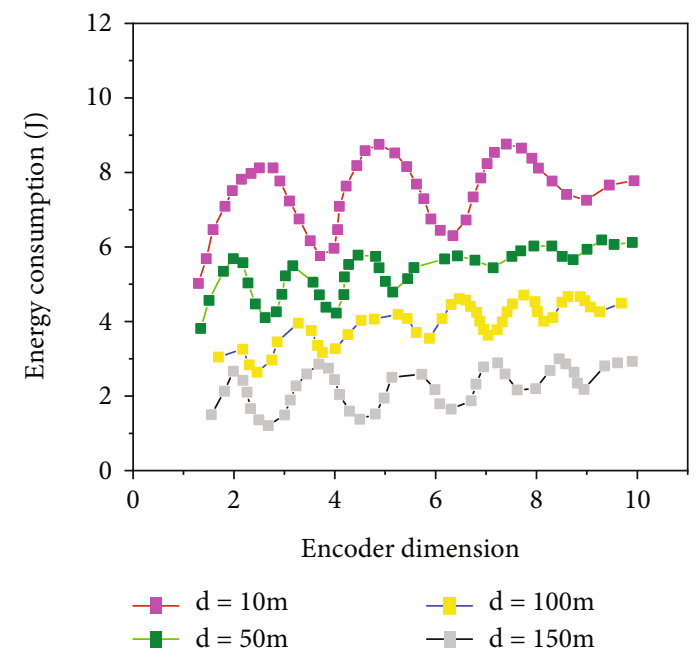

Figure 4: Energy consumption analysis of nodes.

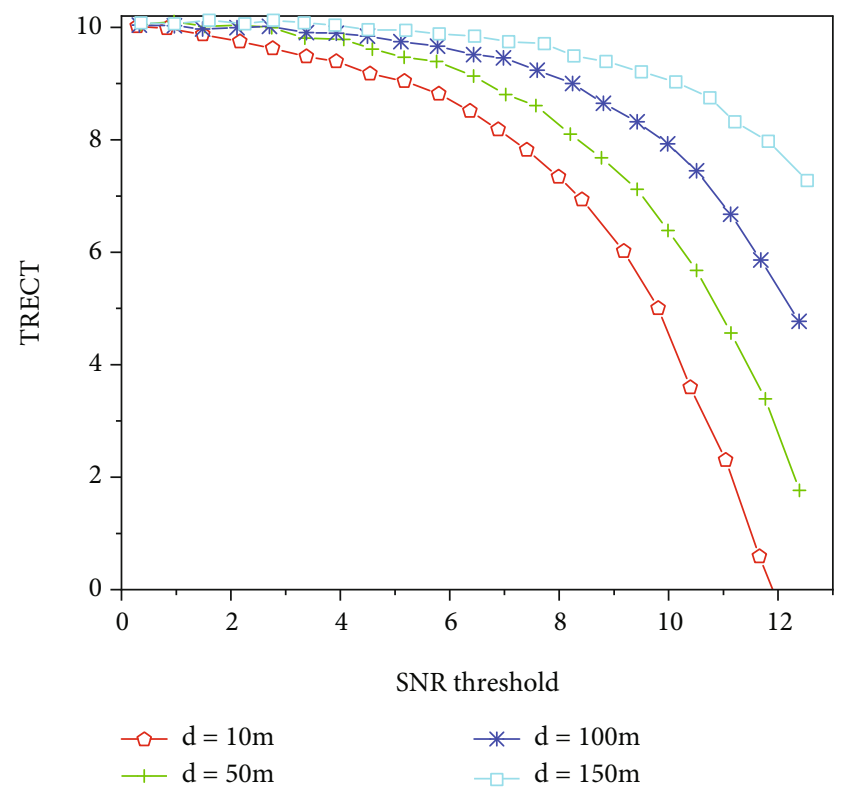

(a) $D_{0}=0.1$

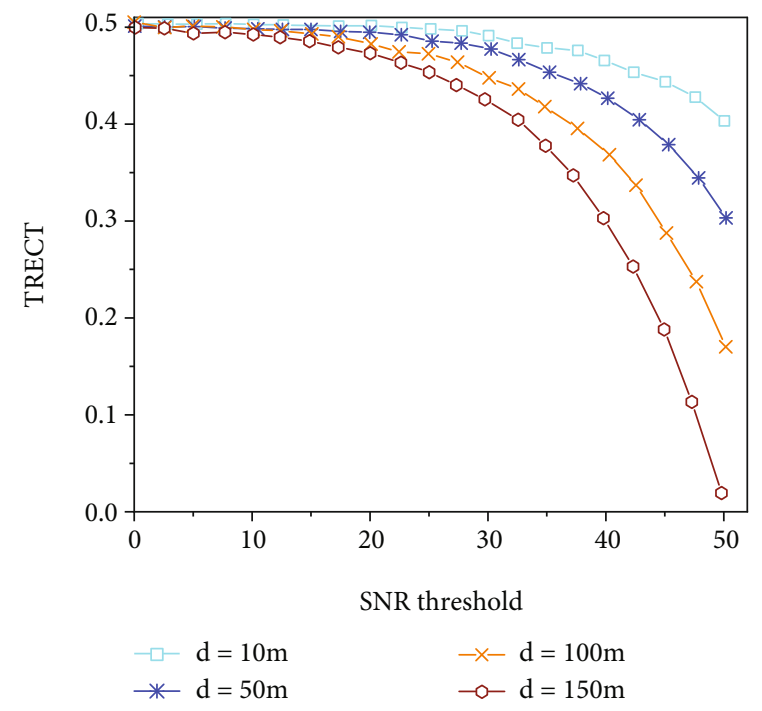

(b) $D_{0}=0.2$

FIgURE 5: Analysis of a TRECT value.

calculations of video coding and decoding per frame, this $r$ decrease is far less than the reduction of transceiver energy consumption.

4.3. Influence of Next Hop Distance. The energy consumption is divided into network energy consumption and node energy consumption. The former refers to the original size of $512 \times 512 \times 8$, the total energy consumed in the whole process from the source node to the sink node, which is the energy consumed by an image node transmitting an image of the same size. The network lifetime is characterized by the number of images that an image node can transmit in an energy acquisition cycle. It is assumed that the energy that a node can collect in a cycle is $10 \mathrm{~J}$. The performance comparison of three different image transmission mechanisms is shown in Figure 6.

As can be seen from Figure 6(a), when the next hop distance is $120 \mathrm{~m}$, the network lifetime of mechanisms transmit 


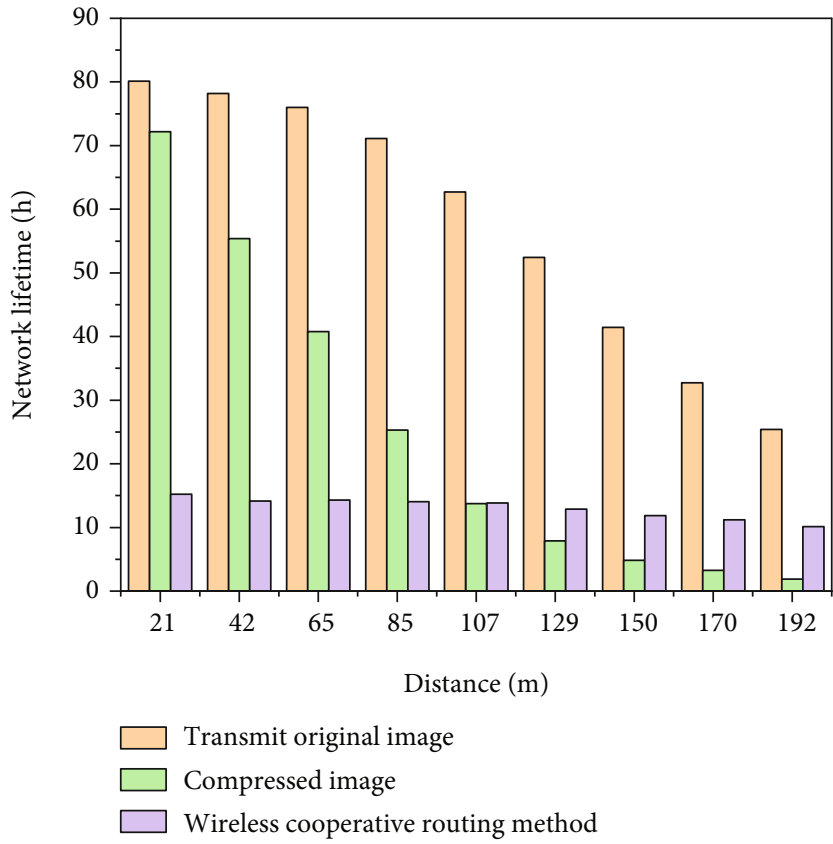

(a) Comparison of network lifetime

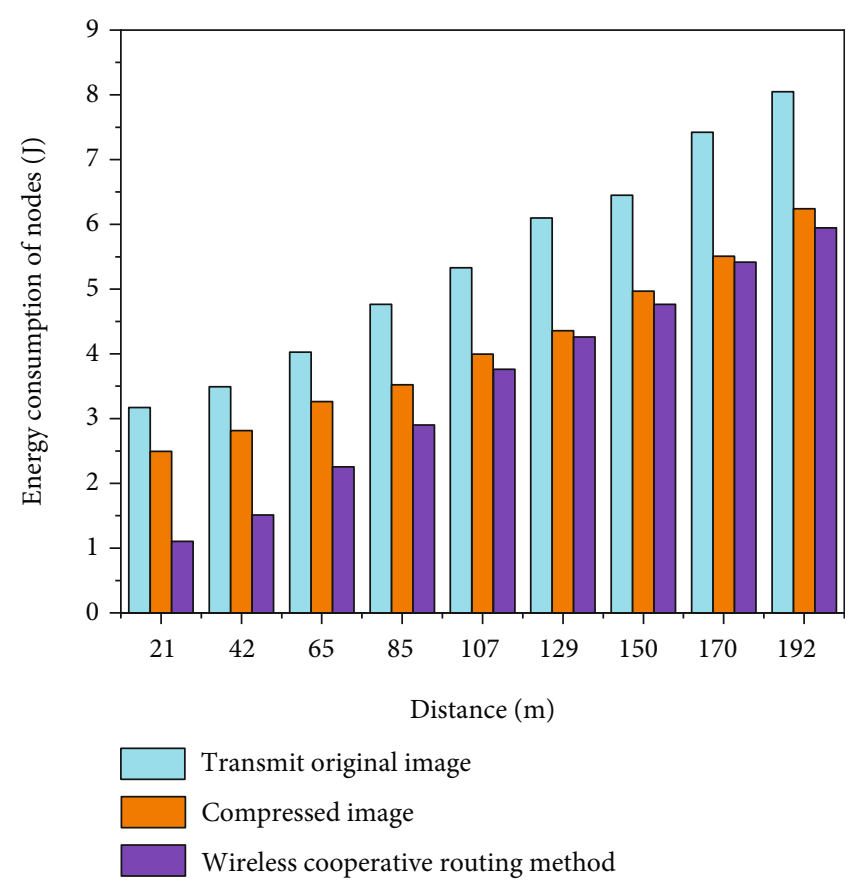

(b) Comparison of node energy consumption

FIGURE 6: Influence of next hop distance.

original image and compressed image is 14 , while that of mechanism, wireless cooperative routing method is 63 , which is 4.5 times that of the first two. With the increasing distance, the proportion of energy consumption of sending images is increasing, and the network lifetime also decreases. When the next hop distance reaches $200 \mathrm{~m}$, the network lifetime of the three mechanisms is 2, 10, and 25 in turn. As can be seen from Figure 6(b), when the next hop distance is small, the energy consumptions of image nodes of transmit original image mechanism and wireless cooperative routing method mechanisms are almost the same, which is less than that of compressed image mechanism; however, with the increase of distance, the energy consumption of mechanism increases sharply, while the energy consumption mechanisms of transmit original image and compressed image increase slowly. It is worth noting that the energy consumption difference between systems transmit original image and compressed image remains unchanged when the distance is large. In fact, the energy consumption of compressing an image using the method in this paper is reduced by $0.4237 \mathrm{~J}$ compared with that directly compressed by the cluster head; that is, the compression energy consumption of image nodes is reduced by $64.5 \%$, and the number of compressed images is 2.82 times that of the latter. In conclusion, regardless of the distance of the next hop, the method proposed in this paper performs best in terms of network lifetime and node energy consumption. It shows that the multihop image transmission mechanism based on image node neighbourhood cooperative compression can effectively reduce the energy consumption of image nodes, balance the network energy consumption, and prolong the network lifetime.
4.4. Influence of Hops and Distance. In simultaneous interpreting, the relationship between the energy consumption of the three transmission mechanisms and the number of hops and distances is shown in Figure 7. In Figure 7(a), $h$ $=2$ and $d_{2}$ increase from $20 \mathrm{~m}$ to $160 \mathrm{~m}$ with step $10 \mathrm{~m}$. In Figure 5(b), $h=3, d_{n}=d_{2}=50 \mathrm{~m}$, and $d_{n}$ increase from $20 \mathrm{~m}$ to $160 \mathrm{~m}$ in steps of $10 \mathrm{~m}$. It can be seen that when the transmission hops and distance are small, the network energy consumption of wireless cooperative routing based on minimum energy consumption is less than that of traditional method 1, but when the transmission hops and distance are large, the network energy consumption of traditional method 1 is slightly higher than that of traditional method 2. The reason is that the image node needs to consume additional energy when distributing the image compression task, and the increased energy consumption is about $0.2328 \mathrm{~J}$. The node energy consumption of wireless cooperative routing based on minimum energy consumption is always less than that of traditional method 1. It can be seen that when the number of hops is large and the distance is long, the traditional method 1 is to increase a small amount of network energy consumption, so as to balance the energy consumption of image clusters and obtain a larger network lifetime.

4.5. Performance Comparison of Different Methods. It can be seen from Figure 8(a) that when the number of hops is $i$ -hop, the network energy consumption of the two mechanisms is almost the same, but when the distance is long, the energy consumption of the method in this paper is slightly higher than that of the latter. This is because in order to be suitable for multihop transmission, the wireless cooperative route based on minimum energy consumption sends 


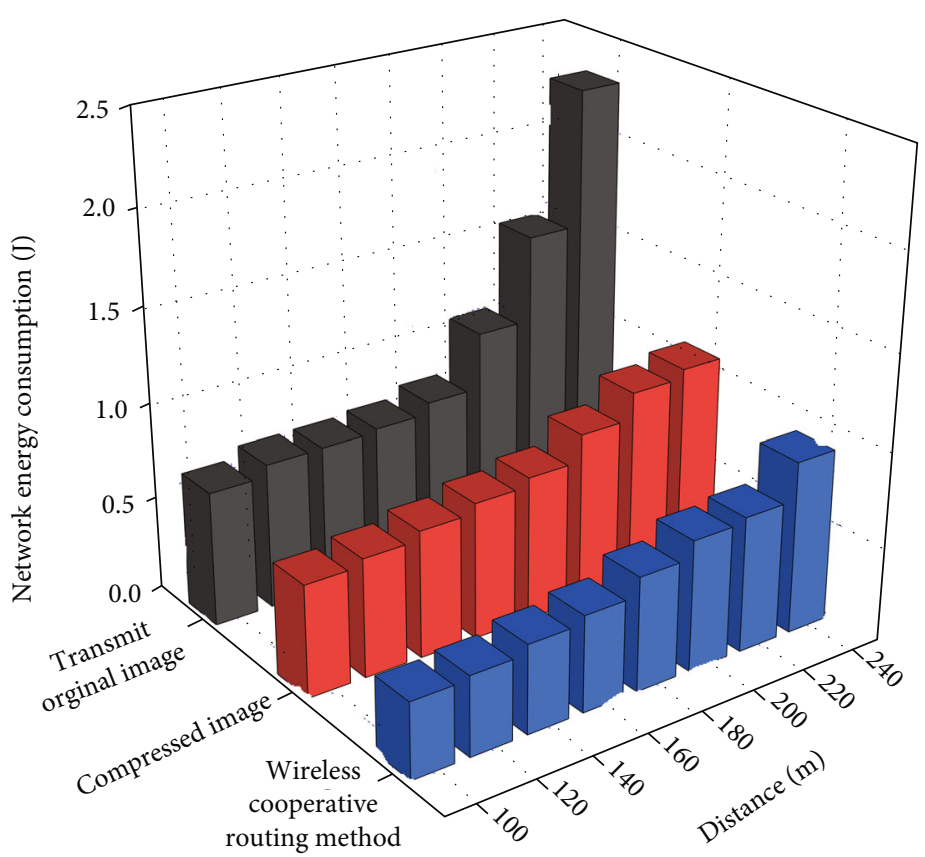

(a) 2-hop network energy consumption

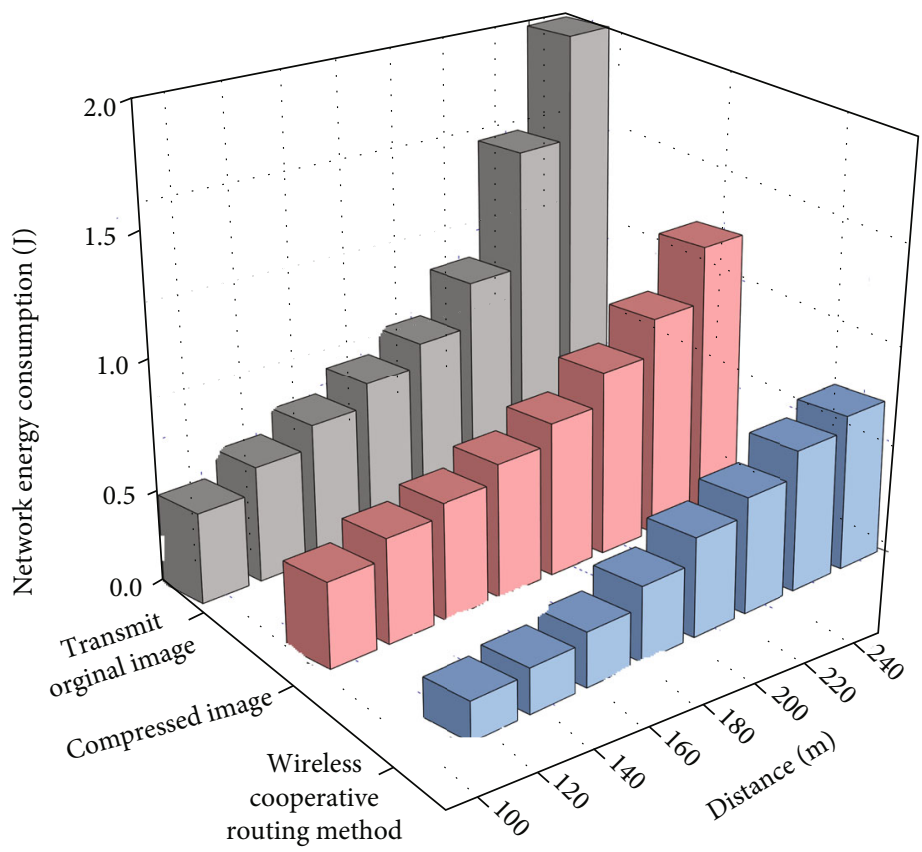

(b) 3-hop network energy consumption

FIgURE 7: Influence of hops and distance.

the image back to the image cluster head after the image compression is completed by the ordinary node, and then, the compressed image is transmitted by the image cluster head. It is not difficult to see from Figures $8(\mathrm{~b})-8(\mathrm{~d})$ that when the number of hops is greater than 1 hop, the network energy consumption of wireless cooperative routing based on minimum energy consumption is lower than the latter, and the more hops and longer transmission distance, the more obvious the energy-saving effect. This is because the wireless cooperative routing based on minimum energy consumption adopts the multihop transmission mechanism based on hops and distance, and the influence of distance and hops from the image node to the BS is fully considered in the image transmission. Therefore, with the increase of distance, the energy consumption will increase sharply. It can be seen that the methods in the literature are only suitable for WMSN with small network coverage, and the wireless cooperative routing based on minimum energy 


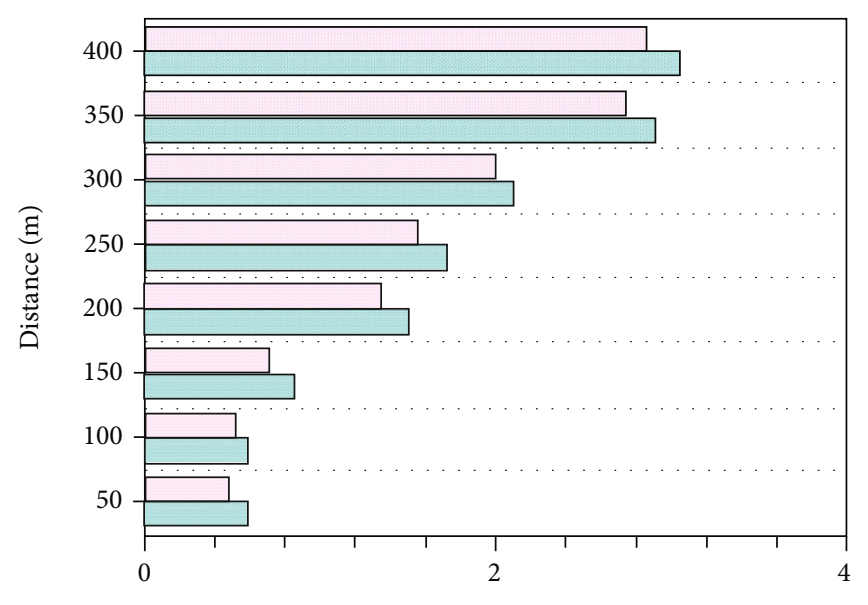

Network energy consumption (J)

Wireless cooprative routing method

2-hop image transmission

(a) 1-hop network energy consumption

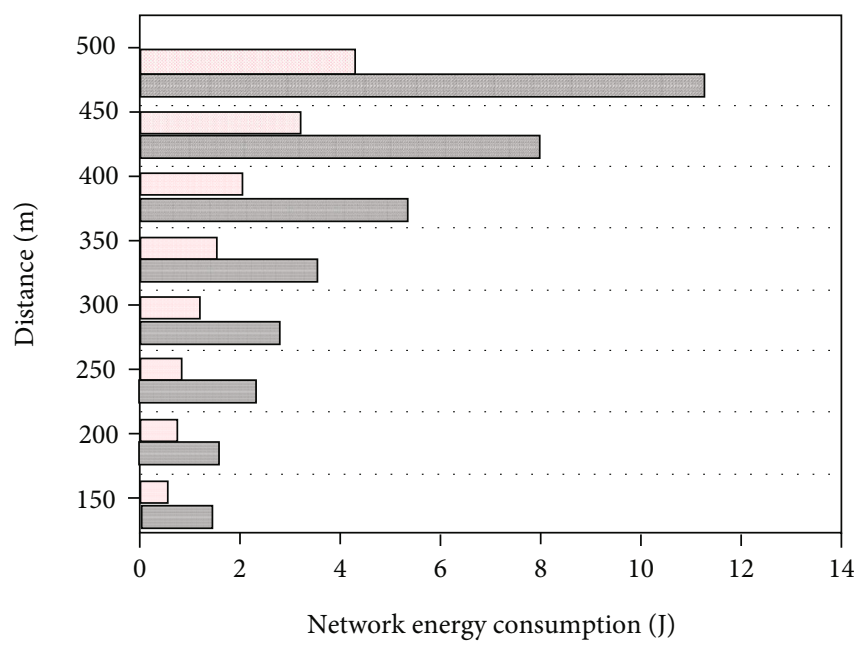

Wireless cooprative routing method

2-hop image transmission

(c) 3-hop network energy consumption

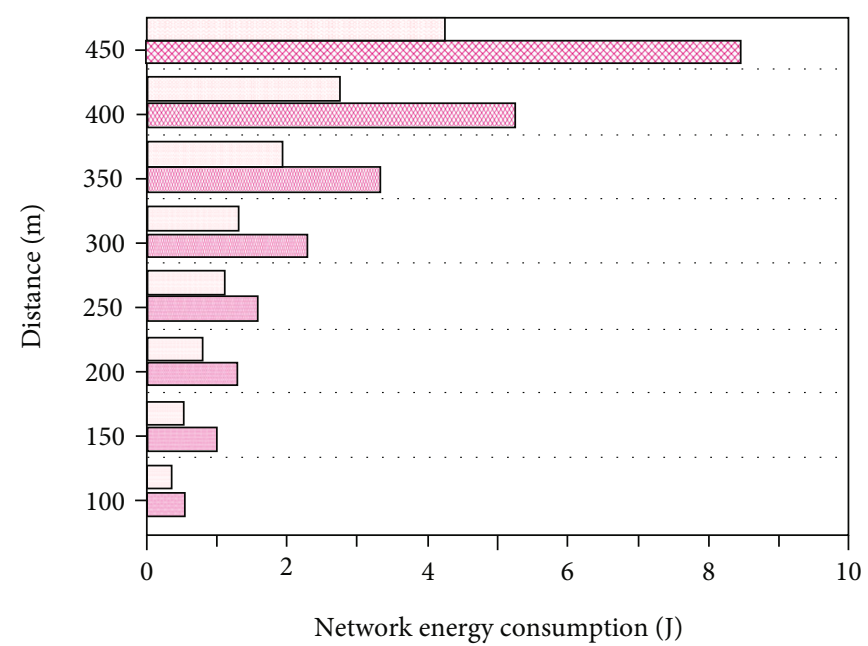

Wireless cooprative routing method

2-hop image transmission

(b) 1-hop network energy consumption

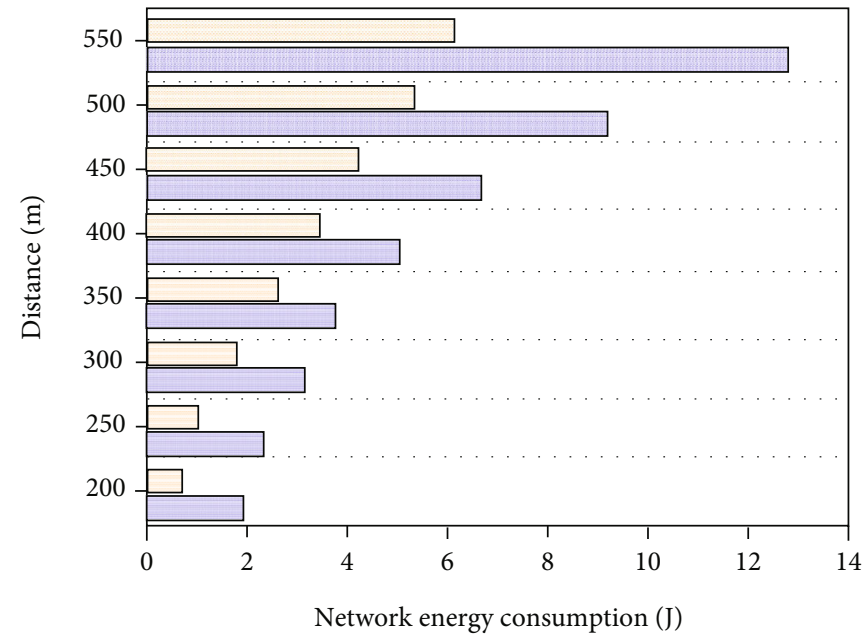

Wireless cooprative routing method

2-hop image transmission

(d) 4-hop network energy consumption

Figure 8: Comparison of energy consumption between two-hop and multihop image transmissions.

consumption is especially suitable for multihop image transmission in large-scale wireless digital media sensor networks.

\section{Conclusion}

In this paper, the energy consumption of digital media art communication is analyzed and studied, and a wireless cooperative routing digital media art communication based on minimum energy consumption is proposed. The energy-saving transmission mode is selected according to the transmission distance and hops. Through the neighbourhood cooperative compression scheme, the network energy consumption is balanced and the energy consumption of image nodes is reduced. The experimental results show that the image node adopts the wireless cooperative routing method based on minimum energy consumption to compress the image, which reduces the energy consumption by $64.5 \%$ and increases the number of compressed images by $182 \%$. Compared with the existing two-hop image transmission mechanism, when the number of hops is 1 hop, the energy consumption is almost the same, but when transmitting images are over a long distance, the network energy consumption of digital media art transmission is significantly lower than the latter. Obviously, combining the multihop image transmission mechanism proposed in this paper with the image compression algorithm with lower energy consumption can undoubtedly further improve the energy efficiency of image nodes, so as to prolong the network lifetime more effectively. In addition, when determining the image transmission path, selecting a highly reliable link can effectively reduce the number of image retransmissions 
and improve the performance of digital media art transmission. Therefore, the next research work is to design a lowenergy image compression algorithm suitable for digital media art communication and a routing protocol based on transmission success rate.

\section{Data Availability}

The data used to support the findings of this study are available from the corresponding author upon request.

\section{Conflicts of Interest}

No potential conflict of interest was reported by the author.

\section{References}

[1] Q. Li, C. Peng, M. Chen et al., "Networked and distributed control method with optimal power dispatch for islanded microgrids," IEEE Transactions on Industrial Electronics, vol. 64, no. 1, pp. 493-504, 2017.

[2] L. M. Candanedo, V. Feldheim, and D. Deramaix, "Data driven prediction models of energy use of appliances in a low-energy house," Energy Buildings, vol. 140, pp. 81-97, 2017.

[3] N. Fei, L. J. Xu, and X. H. Cheng, "Implementation and performance analysis of cooperative routing based on software defined wireless networks," Computer Research and Development, vol. 56, no. 5, pp. 967-976, 2019.

[4] P. J. Wu, F. Gao, C. X. Xing, and L. Luo, "Overview of cooperative routing algorithm design and classification in wireless networks," Communication Technology, vol. 51, no. 6, pp. 1239-1247, 2018.

[5] Z. Y. Yang, "Research on non-cooperative cognitive wireless network routing algorithm based on reinforcement learning," Journal of Xi'an University of Arts and Sciences, vol. 21, no. 1, pp. 68-72, 2018.

[6] J. F. Liu, J. X. Wang, and Z. H. Wang, "Cooperative routing algorithm for multi hop cognitive wireless networks based on game theory," Computer Engineering and Design, vol. 38, no. 5, pp. 1136-1141, 2017.

[7] B. H. Zhang, "On the promotion of digital new media to the dissemination of art information," Science and Technology Communication, vol. 9, no. 8, pp. 64-65, 2017.

[8] L. J. Sun and G. L. Zhang, "Dynamic geographic cooperative routing algorithm for cooperative relay node selection," Computer Engineering and Design, vol. 38, no. 2, pp. 281-286, 2017.

[9] H. Qiao, D. F. Zhang, K. Xie, S. M. He, and J. Zhang, "Joint cooperative routing and channel allocation algorithm in multi-RF wireless mesh networks," Acta Electronica Sinisa, vol. 44, no. 6, pp. 1400-1405, 2016.

[10] L. Zhou, H. Su, H. Tang, and J. H. Han, "Cooperative routing algorithm for wireless networks based on location information," Journal of Electronic Measurement and Instrumentation, vol. 29, no. 5, pp. 708-716, 2015.

[11] H. Qiao, D. F. Zhang, K. Xie, S. M. He, and J. Zhang, "Fair cooperative routing algorithm for distributed multi gateway wireless mesh networks," Journal of Communications, vol. 36, no. 2, pp. 179-189, 2015.

[12] Y. L. Ma and D. L. Hao, "Routing strategy of cross layer cooperation in wireless mesh networks," Modern Electronic Technology, vol. 37, no. 18, pp. 38-40, 2014.
[13] J. Li, "Discussion on the social communication of digital media art in contemporary society," Popular Literature and Art, vol. 12, no. 5, pp. 122-123, 2021.

[14] X. M. Liu, "A review of the research on the digital communication path of regional culture," Northern Media Research, vol. 10, no. 3, pp. 96-100, 2021.

[15] X. Z. Zhao, "Opportunities and challenges faced by the news communication industry from the perspective of digital media technology," News World, vol. 12, no. 1, pp. 57-59, 2021.

[16] C. X. Huang and C. H. Zhu, "Research status, problems and trends of digital media in China," News Forum, vol. 34, no. 6, pp. 20-24, 2020.

[17] A. X. Du, "Research on public domain resources of digital media communication," News Lovers, vol. 17, no. 2, pp. 6668, 2020.

[18] A. H. Xing, "Application of virtual reality technology in digital media design,” Media Forum, vol. 3, no. 24, pp. 45-46, 2020.

[19] F. Li, "Application of digital media technology in the current communication environment," Media Forum, vol. 3, no. 24, pp. 26-27, 2020.

[20] Y. Hao, "Digital media art effectively promotes cultural communication," Marketing, vol. 15, no. 4, pp. 193-194, 2020.

[21] R. Kang, "Analysis of news communication path from the perspective of digital media," China Media Science and Technology, vol. 17, no. 5, pp. 56-58, 2020.

[22] M. Y. Fu and J. H. Xu, "Application of digital media technology in the current communication environment," Drama House, vol. 15, no. 11, pp. 220-223, 2020.

[23] B. Zou, "Research on the communication strategy of traditional culture under the background of new media," Media, vol. 17, no. 9, pp. 90-93, 2019.

[24] Y. Hu, "Promotion of digital media on art communication," Educational Modernization, vol. 6, no. 72, pp. 127-128, 2019. 